\title{
A discovery and redescription of Curubis erratica Simon, 1902 (Araneae: Salticidae) from India
}

\author{
Находка и переописание Curubis erratica Simon, 1902 \\ (Araneae: Salticidae) из Индии
}

\author{
John T.D. Caleb \\ Ажкон Т.А. Камеб \\ Department of Zoology, Madras Christian College, Tambaram, Chennai-600 059, Tamil Nadu, India; email: caleb87woodgate@gmail.com \\ KEY WORDS: Curubis erratica, Salticidae, new record, India, redescription, taxonomy. \\ КЛЮЧЕВЫЕ СЛОВА: Curubis erratica, Salticidae, новая находка, Индия, переописание, таксономия.
}

ABSTRACT. Curubis erratica Simon, 1902 - the type species of the genus Curubis Simon, 1902 - is recorded from India for the first time. The species is redescribed on the basis of Indian specimens, and its female is described for the first time.

РЕЗЮМЕ. Curubis erratica Simon, 1902 - типовой вид рода Curubis Simon, 1902 — отмечается впервые из Индии. Вид переописан на основе индийских экземпляров, и самка данного вида описана впервые.

\section{Introduction}

The genus Curubis Simon, 1902 is a member of the tribe Hasariini of the subfamily Salticinae [Maddison, 2015], and currently consists of the four valid species known from Sri Lanka and India: C. annulata Simon, 1902, C. erratica Simon, 1902, C. sipeki Dobroruka, 2004 and C. tetrica Simon, 1902 [Prószyński, 2015; WSC, 2016]. To date, all the species have been known from the males only. In the present paper, the female of the type species - C. erratica - is described for the first time. Until now, this species has been known from the original description by Simon [1902], except for its later illustrations prepared by Prószyński [1987]. Thus, the present finding is the first formal record of the species in 114 years, after its original description.

\section{Material and methods}

Specimens were examined by means of the Leica S8APO stereoscopic microscope. Photographs of specimens at different views were made by means of the Leica MC120 HD camera and stacked with the Leica Application Suite (LAS) software. The male palp was detached for an examination, while the female copulatory organs were extracted by means of a fine sharp- ened needle and then cleared in $10 \% \mathrm{KOH}$. The nomenclature follows WSC [2016]. The species was identified on the basis of the illustrations provided by Prószyński [1987: 18; 2015]. Leg measurements are given as follows: a total length (femur, patella, tibia, metatarsus, tarsus); all measurements are in millimeters. The studied specimens are deposited in the $\mathrm{Na}$ tional Zoological Collection at the Southern Regional Centre, Zoological Survey of India (ZSI-SRC), Chennai. Abbreviations used in the text: ALE - anterior lateral eye, AME - anterior median eye, PLE - posterior lateral eye, PME — posterior median eye, RTA retrolateral tibial apophysis.

\section{Taxonomy}

\section{Genus Curubis Simon, 1902}

DEFINITION. Small spiders of about $2-4 \mathrm{~mm}$ in length. The male palp with the thick embolus accompanied by the large conductor, the RTA huge and strong (Figs 10, 12, 15) [Żabka, 1988]. The epigyne with a distinctively large caudal lobe protruding beyond the epigastric furrow (Figs 13, 16, 17).

\section{Curubis erratica Simon, 1902}

Figs 1-17, Map.

Curubis erratica Simon, 1902: 372 (D $\left.{ }^{7}\right)$; the holotype is not re-examined.

Curubis erratica: Simon, 1903: 797, fig. 926; Prószyński, 1987: 18 .

MATERIAL. INDIA: $1 \sigma^{7}$ (ZSI-SRC-I/SP 27), Chennai, Thirumullaivoyal $\left(13.125101^{\circ} \mathrm{N}, 80.135654^{\circ} \mathrm{E}\right), 21.73 \mathrm{~m}$ a.s.1., 28 June 2013, J. Caleb; 1 (ZSI-SRC-I/SP 28), same data, 10 October 2013, J. Caleb; 1 ○ (author's personal collection), same locality, 4 January 2016, Kumari; $1 \sigma^{7}$ (author's personal collection), same locality, 10 February 2016, J. Caleb.

DIAGNOSIS. From all its congeners, C. erratica can be distinguished by the thick embolus accompanied by the curved conductor (Figs 11,14) and by the 


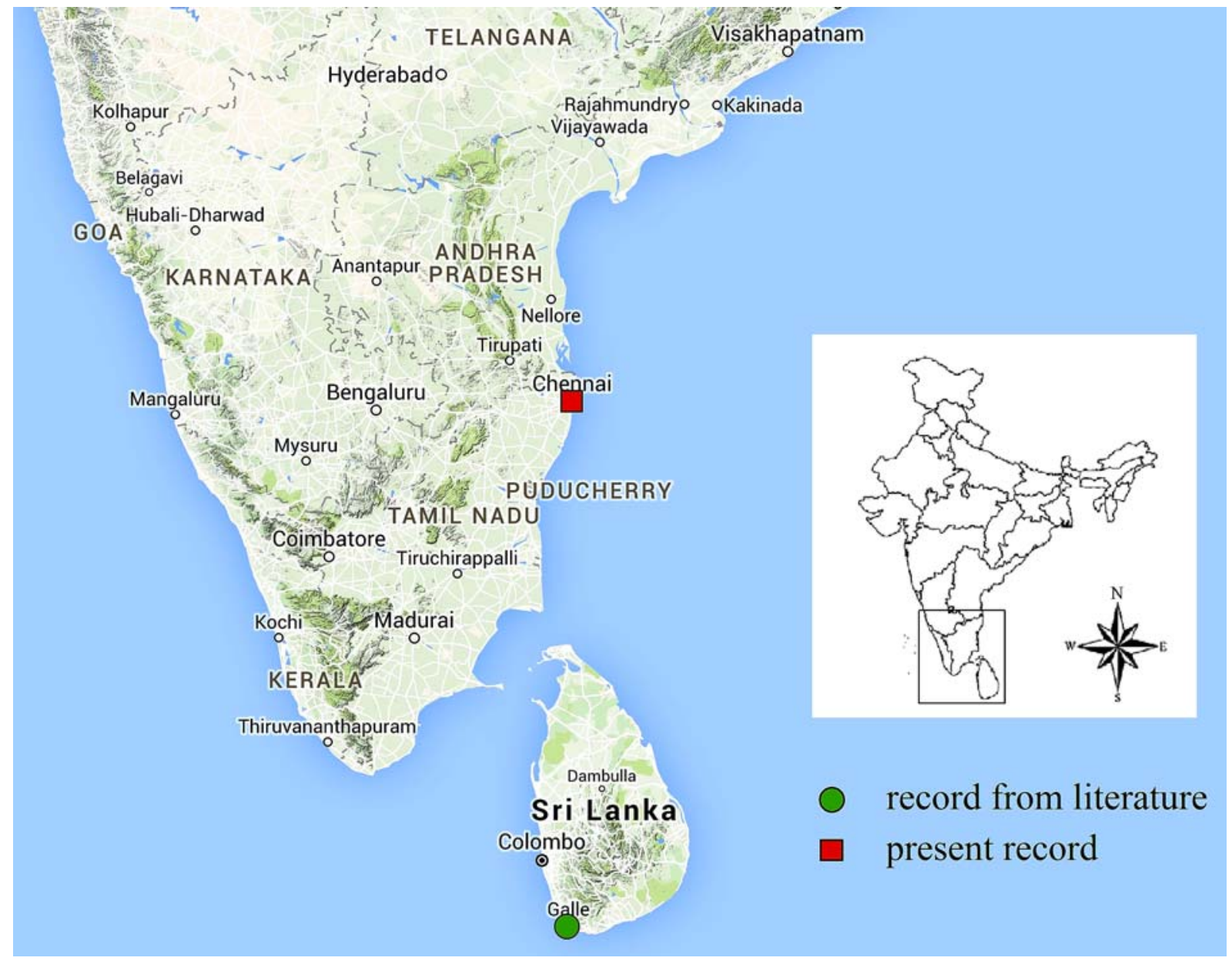

Map. Distributional records of Curubis erratica Simon, 1902.

Карта. Точки распространения Curubis erratica Simon, 1902.

lateral bulbal protrusion near the RTA. RTA is stout and strong, its proximal-dorsal margin with a fin-like protrusion (Figs 10, 12, 14, 15). The epigyne has a wide V-shaped caudal lobe (Figs 13, 16). Insemination ducts make S-shaped twists leading to the spermathecae (Fig. 17).

DISTRIBUTION. Sri Lanka (Galle) and India (Chennai) (see Map).

DESCRIPTION. MALE. Total length 2.86; carapace 1.44 long, 1.83 wide; abdomen 1.42 long, 1.25 wide. Carapace reddish brown, covered with grey hairs (Figs 1, 2). Eyes surrounded by white orbital setae, mixed up with yellowish setae on the anterior margins. Clypeus covered with dense white hairs (Fig. 4). Eye sizes and interdistances: AME 0.35, ALE 0.22, PME 0.09, PLE 0.21; AME-AME 0.04, AME-ALE 0.02, ALE-PME 0.32, PME-PME 1.04, PME-PLE 0.10, PLE-PLE 0.82. Clypeus height 0.21. Chelicerae fissidentate, reddish black; maxillae and labium yellowish, with pale outer edges on the maxillae (Fig. 5). Sternum oval, yellowish, covered with pale yellow hairs (Fig. 3). Leg measurements: I $2.30(0.75,0.59,0.50,0.24$, $0.22)$; II $2.38(0.85,0.43,0.50,0.35,0.25)$; III 2.85
$(0.94,0.59,0.51,0.50,0.31)$; IV $2.92(0.92,0.44$, $0.55,0.51,0.50)$. All legs yellowish, with dark annular rings at segment joints; all femora with dark proximal halves. Abdomen rounded, covered with grey hairs. Dorsum with a pair of yellowish spots anteriorly and two faint chevron shaped markings medially (Figs 1, 2). Venter brownish, with yellow spots. Spinnerets brownish yellow. Palp yellowish brown, dorsally covered with greyish white hairs; palpal femur and patella dorsally covered with white hairs (Figs 4, 10). Embolus thick, bulbus broad (Figs 11, 14), RTA stout, long, slightly bending ventrad, its proximal-dorsal margin with a fin-shaped protrusion (Figs 10-12).

FEMALE. Total length 3.72; carapace 1.90 long, 1.37 wide; abdomen 1.82 long, 1.45 wide. Eye sizes and interdistances: AME 0.36, ALE 0.23, PME 0.08, PLE 0.21; AME-AME 0.03, AME-ALE 0.04, ALEPME 0.31, PME-PME 1.01, PME-PLE 0.16, PLEPLE 0.82 , clypeus height 0.09 . Leg measurements: I $2.59(0.89,0.54,0.45,0.40,0.31)$; II $2.36(0.87,0.50$, $0.45,0.33,0.21)$; III $2.81(1.15,0.38,0.31,0.61,0.36)$; IV $2.99(0.98,0.40,0.64,0.59,0.38)$. Coloration similar to that of the male. Epigyne with a large and wide, 

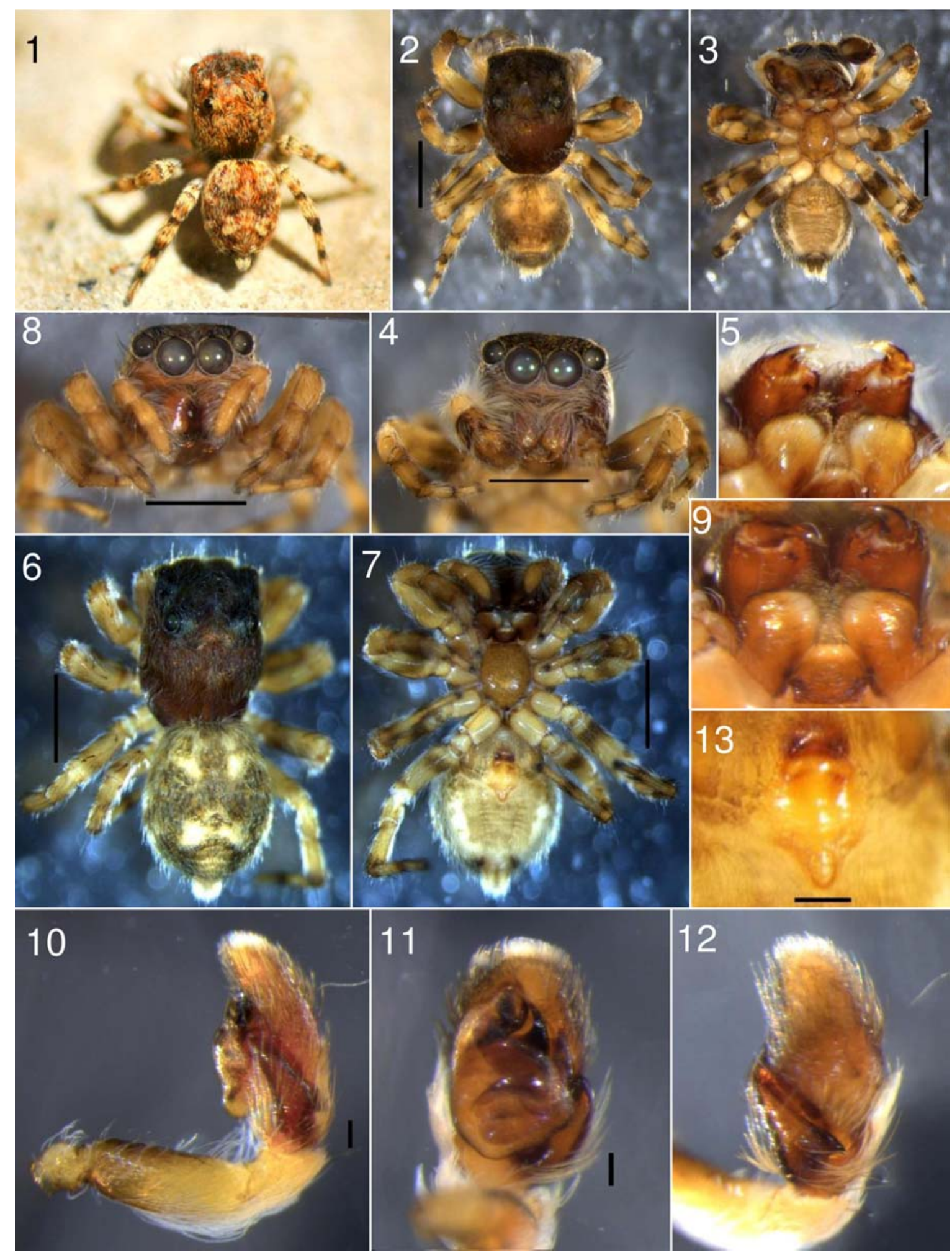

Figs 1-13. General appearance and copulatory organs of Curubis erratica Simon, 1902: 1, 2 - male, dorsal view; 3 - ditto, ventral view; 4 - ditto, front view; 5 - male chelicerae, ventral view; 6 - female, dorsal view; 7 - ditto, ventral view; 8 - ditto, front view; 9 - female chelicerae, ventral view; 10 - male palp, retro-lateral view; 11 - ditto, ventral view; 12 - ditto, dorso-lateral view; 13 epigyne, ventral view. Scale bars: $1 \mathrm{~mm}(2-4,7-8) ; 0.1 \mathrm{~mm}(10-13)$.

Рис. 1-13. Общий вид и копулятивные органы Curubis erratica Simon, 1902: 1, 2 - самец, дорзально; 3 - тоже, вентрально; 4 - тоже, вид спереди; 5 - хелицеры самца, вентрально; 6 - самка, дорзально; 7 - тоже, вентрально; 8 - тоже, вид спереди; 9 - хелицеры самки, вентрально; 10 - пальпа самца, ретро-латерально; 11 - тоже, вентрально; 12 - тоже, дорзо-латерально; 13 - эпигина, вентрально. Масштаб: 1 мм (2-4, 7-8); 0,1 мм (10-13). 

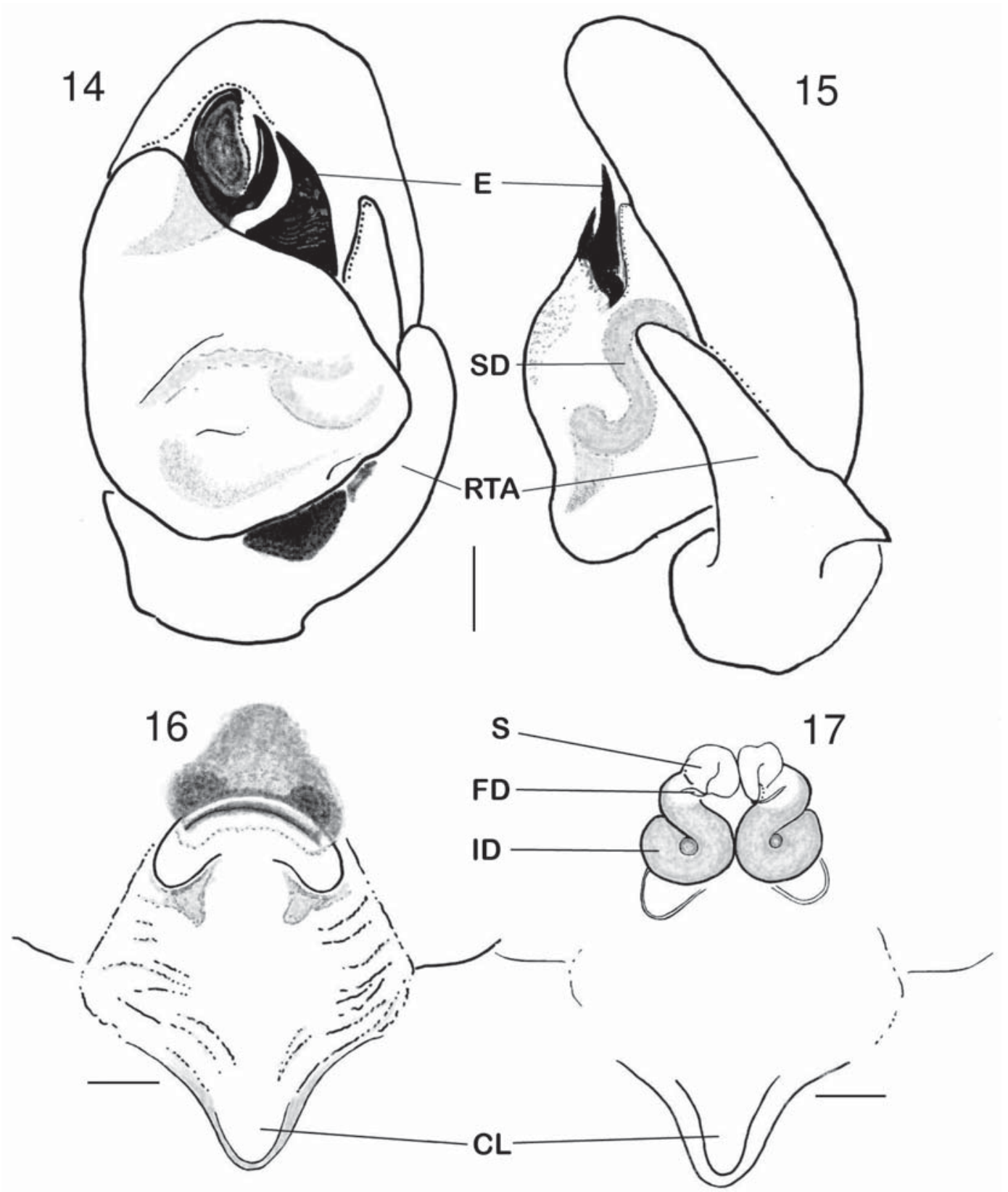

Figs 14-17. Copulatory organs of Curubis erratica Simon, 1902: 14 - male palp, ventral view; 15 - ditto, retro-lateral view; 16 epigyne, ventral view; 17 - spermathecae, dorsal view. Scale bars: $0.1 \mathrm{~mm}$. Abbreviations: CL - caudal lobe, E - embolus, FD fertilization duct, ID - insemination duct, RTA — retrolateral tibial apophysis, S — spermathecae, SD — sperm duct.

Рис 14-17. Копулятивные органы Curubis erratica Simon, 1902: 14, пальпа самца, вентрально; 15 - тоже, ретро-латерально; 16 - эпигина, вентрально; 17 - сперматека, дорзально. Масштаб: 0.1 мм. Сокарщения: CL — каудальная доля, Е — эмболюс, FD - оплодотворительный канал, ID - совокупительный канал, RTA - ретролатеральный отросток, S - сперматека, SD семенной каналец.

V-shaped sclerotized caudal lobe; epigynal fold arched (Figs 13, 16). Insemination ducts are heavily sclerotized near the copulatory openings, ducts make S-shaped twists (Fig. 17).

ACKNOWLEDGEMENTS. Special thanks go to Prof Jerzy Prószyński (Siedlce, Poland) for his encouragement and valuable suggestions. I wish to thank
Dr Krushnamegh Kunte, Lab 8, National Centre for Biological Sciences (NCBS), Bangalore for allowing me to use the stereo microscope facility. Thanks also go to Dr Ilango, Officer-in-Charge, Southern Regional Centre, ZSI, Chennai, for helping me to deposit the specimens. Finally, Dr Dmitri Logunov (Manchester, UK) is thanked for his editorial and linguistic help. 


\section{References}

Maddison W.P. 2015. A phylogenetic classification of jumping spiders (Araneae: Salticidae) // Journal of Arachnology. Vol.43. P.231-292.

Prószyński J. 1987. Atlas rysunków diagnostycznych mniej znanych Salticidae 2 // Zeszyty Naukowe Wyższej Szkoly RolniczoPedagogicznej v Siedlcach. 172 pp.

Prószyński J. 2015. Salticidae (Araneae) of the world, online at: http://www.peckhamia.com/salticidae/ (accessed on 2nd February, 2016).
WSC 2016. World Spider Catalog. Natural History Museum Bern, online at: http://wsc.nmbe.ch,version 17 (accessed on 3rd March 2016).

Simon E. 1902. Description d' Arachnides nouveaux de la famille des Salticidae (Attidae) (suite) // Annales de la societe entomologique de Belgique, Bruxelles. Vol.46. P.24-56, 363-406. Simon E. 1903. Histoire naturelle des araignées. Paris. T.2. P.6691080

Żabka M. 1988. Salticidae (Araneae) of Oriental, Australian and Pacific Regions III // Annales zoologici, Warszawa. Vol.41. No.14. P.421-479.

Responsible editor D.V. Logunov 